\title{
Interdisciplinary Management of Visceral Artery Aneurysms and Visceral Artery Pseudoaneurysms
}

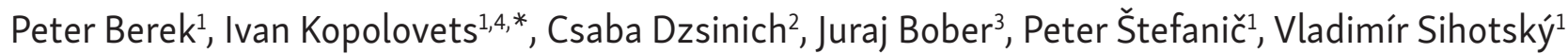

\begin{abstract}
The paper presents the results of treating 14 patients, namely eight patients with visceral artery aneurysms and six patients with visceral artery pseudoaneurysms. In $64.3 \%$ of the patients, the initial diagnosis was made based on the ultrasound examination. All the patients (100\%) underwent CT angiography, while angiography was performed in $71.4 \%$ of the cases. Five (35.7\%) patients with visceral artery pseudoaneurysms were emergently hospitalized; among them, the signs of bleeding were observed in 2 patients. In 9 patients, pathology was detected during tests for other conditions. Five (35.7\%) patients underwent endovascular treatment, while 9 (64.3\%) patients received surgical treatment. Endovascular interventions and open surgery demonstrated a nil mortality rate. After endovascular treatment, stent thrombosis was found in 1 patient. In the case of surgical treatment, visceral artery aneurysm was observed in 1 patient who underwent the resection of superior mesenteric artery pseudoaneurysm.

Conclusions. The choice of the method of treating visceral artery aneurysms and visceral artery pseudoaneurysms depends on the location, size, anatomic features of the visceral arteries and the clinical course of the disease. Both endovascular and surgical treatment demonstrate good postoperative outcomes. Visceral ischemia is one of the most serious complications in the postoperative period, which can complicate both the diagnosis and the choice of treatment tactics.
\end{abstract}

\section{KEYWORDS}

visceral artery pseudoaneurysm; visceral artery aneurysms; rupture risk; hemorrhage; visceral ischemia

AUTHOR AFFILIATIONS

${ }^{1}$ Clinic of Vascular Surgery, East Slovak Institute of Cardiovascular Diseases, Faculty of Medicine, Pavol Jozef Šafárik University, Košice, Slovak Republic

${ }^{2}$ Department of Cardiovascular and Thoracic Surgery at the National Institute of Health of Hungary, Budapest

${ }^{3}$ 1st Department of Surgery, Pavol Jozef Šafárik University, Faculty of Medicine, Košice, Slovak Republic

${ }^{4}$ Uzhhorod National University, Medical Faculty, Department of Surgical Diseases, Uzhhorod, Ukraine

* Corresponding author: Clinic of Vascular Surgery, East Slovak Institute of Cardiovascular Diseases, Ondavská 8, 04001 Košice, Slovak Republic; e-mail: i.kopolovets@gmail.com

Received: 5 July 2019

Accepted: 5 August 2019

Published online: 18 May 2020

Acta Medica (Hradec Králové) 2020; 63(1): 43-48

https://doi.org/10.14712/18059694.2020.14

(c) 2020 The Authors. This is an open-access article distributed under the terms of the Creative Commons Attribution License (http://creativecommons.org/licenses/by/4.0), which permits unrestricted use, distribution, and reproduction in any medium, provided the original author and source are credited. 


\section{INTRODUCTION}

Visceral artery aneurysms and pseudoaneurysms are rare yet serious vascular lesions that are quite difficult to diagnose. According to literature, visceral aneurysms account for $2-3 \%$ of all the cases of vascular aneurysms $(1,2)$. Risk factors for visceral aneurysm development include atherosclerosis, inflammatory conditions within the abdominal cavity and the retroperitoneal space, portal hypertension, connective tissue diseases, whereas visceral artery pseudoaneurysms are caused by destructive pancreatitis, iatrogenic injury to the visceral arteries, abdominal trauma $(3,4)$. The most common visceral artery aneurysm localization is as follows: the splenic artery $-60 \%$, the common hepatic artery $-20 \%$, the gastroduodenal artery $-6 \%$, the superior mesenteric artery $-5.5 \%$, the celiac artery $-4.5 \%$, other arteries $-4 \%$. The risk of visceral aneurysm rupture ranges from $5 \%$ for aneurysms that are $15-20 \mathrm{~mm}$ in diameter to $50-70 \%$ for aneurysms with diameters greater than $30 \mathrm{~mm}(5,6)$. According to the international recommendations, endovascular or surgical treatment is indicated for the patients with visceral aneurysm larger than $20 \mathrm{~mm}$, whereas, due to a high rupture risk, surgery is indicated for every patient with pseudoaneurysm $(1,7,8)$. With the development of interventional radiology, endovascular methods of treating visceral artery aneurysms and pseudoaneurysms are implemented into practice $(9,10)$; however, traditional surgical treatment remains relevant, especially in the cases when it is impossible to perform the endovascular treatment $(2,11,12)$. Considering a rarity of this pathology, specifics of its diagnosis and treatment, we have decided to share our own experience of treating visceral artery aneurysms and pseudoaneurysms.

\section{MATERIAL}

The paper presents the results of treating 14 patients during 2008-2018. Among them, there were 8 (57.1\%) females and $6(42.9 \%)$ males. The youngest patient was 47 years old; the oldest patient was 78 years old. According to the patients' past medical history, five of them underwent abdominal surgery (gastrectomy - 1 patient; pancreatoduodenal resection - 1 patient; pancreatic cyst drainage - 2 patients; surgical revision of the abdominal cavity in multi-trauma, splenectomy - 1 patient). All the patients underwent ultrasound examination of the abdominal cavity and the visceral arteries, enhanced CT angiography. Angiography of the aorta and the visceral arteries was applied in 10 cases. By the clinical course, five patients with complaints of severe epigastric pain and general weakness were emergently hospitalized, while nine patients complained of dull epigastric pain and epigastric heaviness.

\section{RESULTS}

DIAGNOSIS OF VISCERAL ARTERY ANEURYSMS AND PSEUDOANEURYSMS

In $9(64.3 \%)$ out of 14 patients, the initial diagnosis was made based on ultrasound examination. Using angiogra- phy and CT angiography, a differential diagnosis between visceral artery aneurysm and visceral artery pseudoaneurysm was made, and the size of pathological formation was determined. The localization of visceral artery aneurysms and pseudoaneurysms is presented in Table 1.

Tab. 1 Visceral artery aneurysm localization.

\begin{tabular}{|l|c|c|}
\hline Visceral artery & $\begin{array}{l}\text { Visceral artery } \\
\text { aneurysm }\end{array}$ & $\begin{array}{l}\text { Visceral artery } \\
\text { pseudoaneurysm }\end{array}$ \\
\hline Splenic artery & 3 & 3 \\
\hline $\begin{array}{l}\text { Superior mesenteric } \\
\text { artery }\end{array}$ & 1 & 2 \\
\hline Celiac artery & 1 & 1 \\
\hline Common hepatic artery & 1 & - \\
\hline $\begin{array}{l}\text { Pancreaticoduodenal } \\
\text { artery }\end{array}$ & 1 & - \\
\hline Left gastric artery & 1 & - \\
\hline
\end{tabular}

The smallest visceral artery aneurysm diameter was $23 \times 27 \mathrm{~mm}$, while the greatest one was $65 \times 72 \mathrm{~mm}$ (Fig. 1).

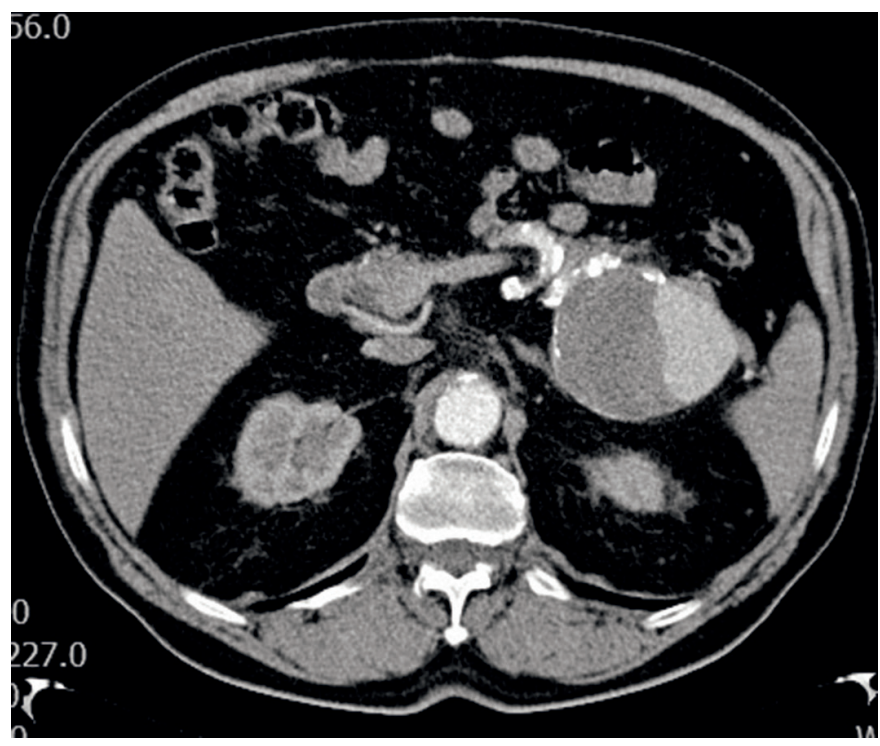

Fig. 1 CT angiography scan of splenic artery aneurysm with a diameter of $72 \times 65 \mathrm{~mm}$.

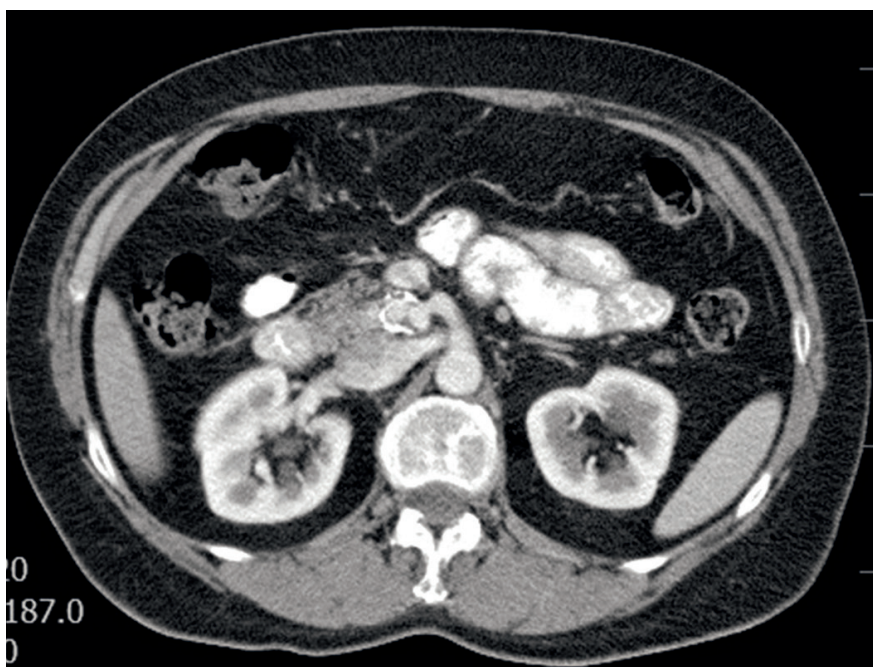

Fig. 2 CT angiography scan of superior mesenteric artery pseudoaneurysm with a diameter of $25 \times 20 \mathrm{~mm}$. 
The smallest diameter of visceral artery pseudoaneurysm was $25 \times 20 \mathrm{~mm}$, while the greatest one was $52 \times 25 \mathrm{~mm}$ (Fig. 2).

Multiple aneurysms in the splenic artery were detected in 1 patient. In 1 patient, in addition to splenic artery aneurysm, infrarenal aortic artery aneurysm with a diameter of $62 \times 58 \mathrm{~mm}$ was found. In 1 case, celiac artery aneurysm extended to the hepatic artery (Fig. 3).

\section{TREATMENT OF VISCERAL ARTERY ANEURYSMS} AND PSEUDOANEURYSMS

Five (35.7\%) patients received endovascular treatment. Endovascular treatment tactics were determined by both vascular surgeon and interventional radiologist. Embolization was used in 2 patients with pseudoaneurysm of the distal part of the splenic artery.

One patient with an aneurysm of the proximal part of the splenic artery underwent coil occlusion. Endovascular aneurysm repair was performed in 1 patient with celiac artery pseudoaneurysm and one patient with common hepatic artery aneurysm.

In other cases $(64.3 \%)$, surgical treatment was preferred: ligature of the splenic artery - 1 patient; resection of splenic artery aneurysm with direct end-toend anastomosis - 2 patients; resection of celiac artery aneurysm and celiac artery reconstruction with a polytetrafluoroethylene (PTFE) graft - 1 patient (Fig. 4); resection of pancreaticoduodenal artery aneurysm and direct suture of the pancreaticoduodenal artery - 1 patient; resection of left gastric artery aneurysm with left gastric artery plasty - 1 patient; resection of superior mesenteric artery aneurysm with vein plasty - 1 patient; resection of superior mesenteric artery pseudoaneurysm with direct sutures - 2 patients.

Endovascular interventions and open surgery demonstrated a nil mortality rate. After endovascular treatment, stent thrombosis was found in 1 patient. After surgical

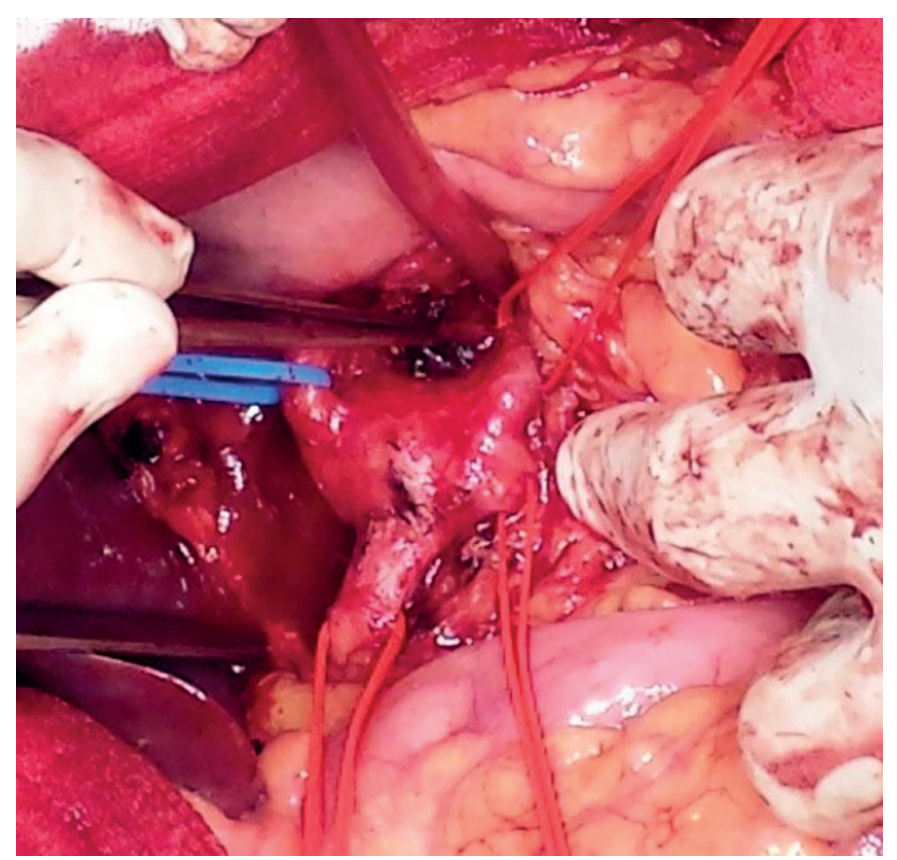

Fig. 3 Celiac artery aneurysm (Intraoperative image). treatment in the early postoperative period, serious complication (visceral artery aneurysm) was observed in 1 patient who underwent the resection of superior mesenteric artery pseudoaneurysm.

Considering a particular interest of this case report, we propose its more detailed presentation.

A 57-year-old female patient with complaints of general weakness, mild epigastric pain, and diarrhea were hospitalized to the Department of Surgery No 1. According to past medical history, the patient had undergone pancreaticoduodenal resection for pancreatic head tumor three weeks prior hospitalization. According to the surgical treatment protocol, pancreatic tumor infiltrated the adventitia of the anterior surface of the superior mesenteric artery that required the preparation of malignant pancreatic formation at the subadventitial layer of the superior mesenteric artery. The pancreatic tumor was removed ad block without any signs of intraoperative bleeding.

At the hospitalization stage, abdominal ultrasound was performed that revealed the collection of hypoechoic fluid $5 \times 2.5 \times 1.5 \mathrm{~cm}$ in size in the epigastric region. To specify the diagnosis, there was prescribed CT angiography of the abdominal cavity, that, during the arterial phase of contrast administration, revealed a rounded area of contrast medium accumulation $50 \times 42 \mathrm{~mm}$ in size to the left of the aorta and approximately $1.5-2 \mathrm{~cm}$ below the origin of the superior mesenteric artery.

The results obtained confirmed superior mesenteric artery pseudoaneurysm (Fig. 5).

The interventional radiologist did not recommend endovascular treatment of pseudoaneurysm as, according to CT angiography, significant anatomical and topographical changes in the superior mesenteric artery were observed.

Taking into account the presence of superior mesenteric artery pseudoaneurysm confirmed by CT angiography, the vascular surgeon recommended surgical resection of superior mesenteric artery pseudoaneurysm. The patient underwent elected laparotomy. During surgical revision,

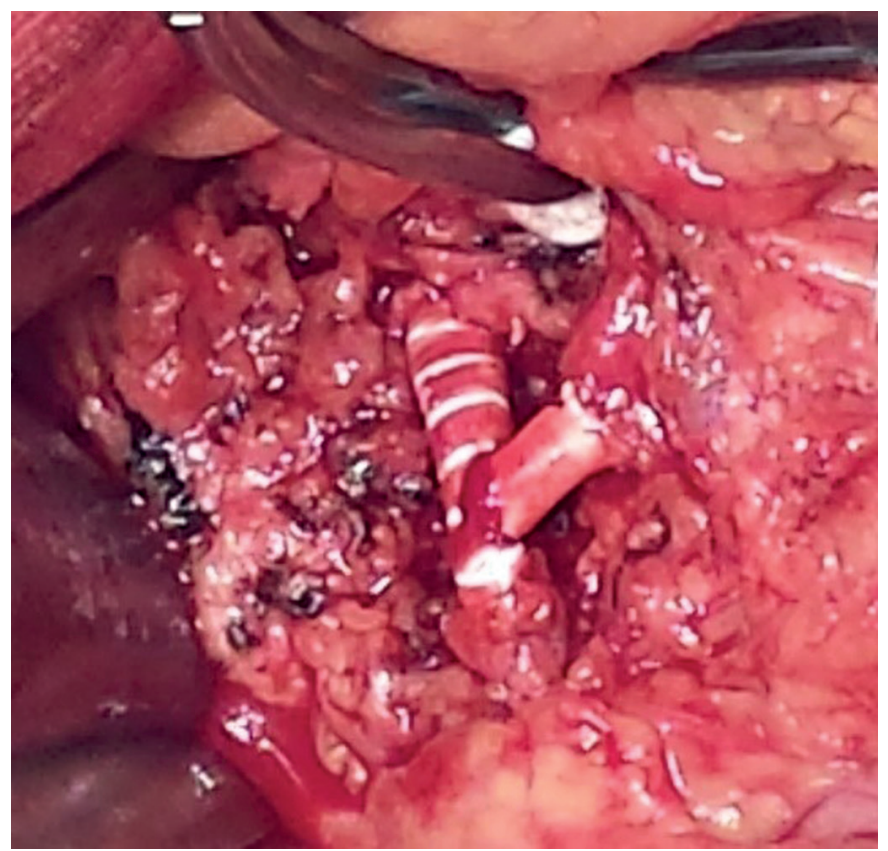

Fig. 4 Arterial reconstruction of the celiac artery with a PTFE Graft. 
in the projection of the superior mesenteric artery, a pulsating mass $5 \times 4 \mathrm{~cm}$ in diameter was detected. The superior mesenteric artery was prepared proximally and distally to the pseudoaneurysm. After injection of 5,000 units of heparin and clamping (compression) of the superior mesenteric artery, pseudoaneurysm was mobilized on the anterior surface of the superior mesenteric artery, where a $2-3-\mathrm{mm}$ opening was found. Considering a pronounced subacute process, we have decided to perform minimally invasive surgery. Namely three single transverse sutures were applied to the superior mesenteric artery. After blood flow restoration, excellent pulsation of the superior mesenteric artery distal to sutures was detected.

However, the patient's clinical condition in the postoperative period was complicated; diffuse abdominal pain and weakly positive signs of peritoneal irritation were observed on the second day after surgery. CT angiography of the abdomen was emergently performed that revealed superior mesenteric artery occlusion. Relaparotomy and surgical revision were urgently performed, taking into account acute intestinal ischemia. Superior mesenteric artery occlusion at the site of pseudoaneurysm resection was found. Arterial reconstruction has been decided to be performed: iliac-mesenteric bypass with the great saphenous vein (Fig. 6).

As it was impossible to assess the viability of the small intestine, the abdomen was left open for eight hours for planned second-look reoperation; then, the small intestine was surgically revised. There was found the necrotic segment of the jejunum. There was performed segmental resection of the jejunum $(70-80 \mathrm{~cm})$. The postoperative course was uncomplicated. There were prescribed anti-inflammatory, detoxification, and antiplatelet therapies.

\section{DISCUSSION}

With the implementation of diagnostic methods such as angiography and CT angiography into clinical practice, visceral artery aneurysms and pseudoaneurysms can be detected before their complications develop that, certainly, increases the quality of treatment thereby reducing the risk of postoperative complications $(8,13)$.

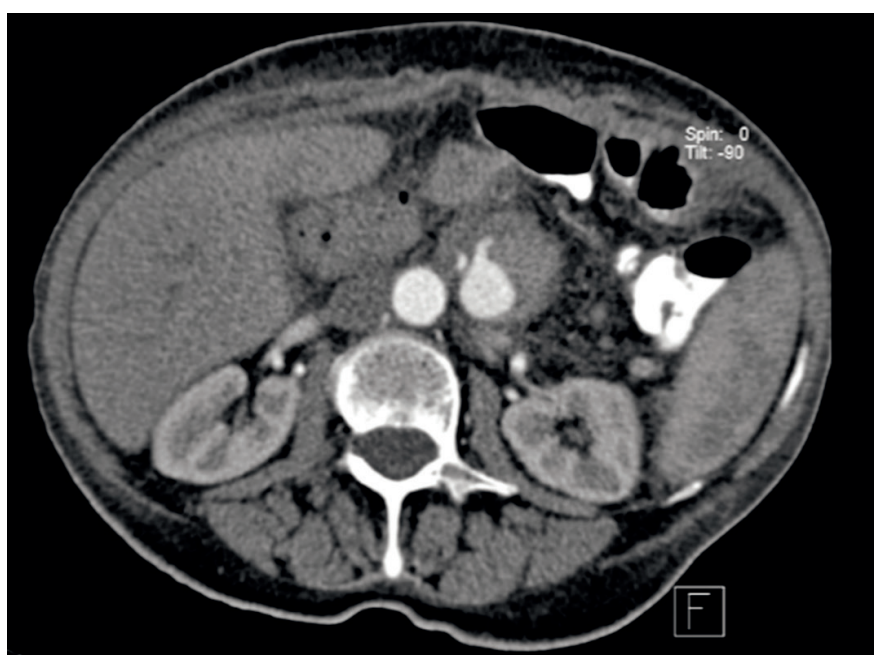

Fig. 5. CT scan of superior mesenteric artery pseudoaneurysm
Digital angiography is currently the gold standard for diagnosing pseudoaneurysms as it allows real-time assessment of the site of extravasation. Digital angiography has the highest sensitivity (100\%), followed by CT (67\%) and ultrasound (50\%) (14). In our study, the initial diagnosis of visceral artery aneurysm based on ultrasound examination was made in $64.3 \%$ of the patients; all the patients (100\%) underwent CT angiography, while angiography was performed in $71.4 \%$ of the cases.

The ratio of visceral artery aneurysms to visceral artery pseudoaneurysms depends on visceral artery location. The most common visceral artery aneurysm localization is the splenic artery while pseudoaneurysms of the gastroduodenal and superior mesenteric arteries are more common as compared to their aneurysms ( $89 \%$ vs. $11 \%$ and $67 \%$ vs. $33 \%$ respectively) $(2,9)$.

More than $60 \%$ of visceral artery pseudoaneurysms occur secondary to pancreatitis, and almost $10-17 \%$ of pseudocysts in patients with chronic pancreatitis are complicated by the development of visceral artery pseudoaneurysms (15).

Blunt or penetrating abdominal trauma and iatrogenic injury after hepatobiliary or vascular surgery, or pancreatic head biopsy may result in visceral artery pseudoaneurysm as well $(7,13)$.

Approximately $80 \%$ of visceral artery aneurysms are asymptomatic being detected during tests for other conditions. Almost $20 \%$ of visceral artery aneurysms have severe clinical manifestations; in $9 \%$ of the cases, they result in death (2). Clinical manifestations of visceral artery aneurysms are non-specific. The patients complain of abdominal discomfort and abdominal pain that is not related to food intake. At the same time, in most patients with visceral artery pseudoaneurysms, the symptomatic clinical course is found; they complain of abdominal and epigastric pain; hematemesis and melena may be observed (3). Among 14 patients, 5 (35.7\%) individuals with visceral artery pseudoaneurysms were emergently hospitalized; among them, the signs of bleeding were observed in 2 patients. In 9 patients, pathology was detected during tests for other conditions; among them, three patients with recurrent symptoms of chronic pancreatitis were hospitalized in the surgical department.

The choice of the method for treatment of visceral artery aneurysms and pseudoaneurysms remains controversial, and the prognostic indicators of the clinical course depend on many factors, namely aneurysm localization and size, clinical manifestations, work experience of the surgical team and technical capabilities of a healthcare institution (7).

The method of choice should be endovascular treatment (selective embolization, coils, stent, gelatin foam,

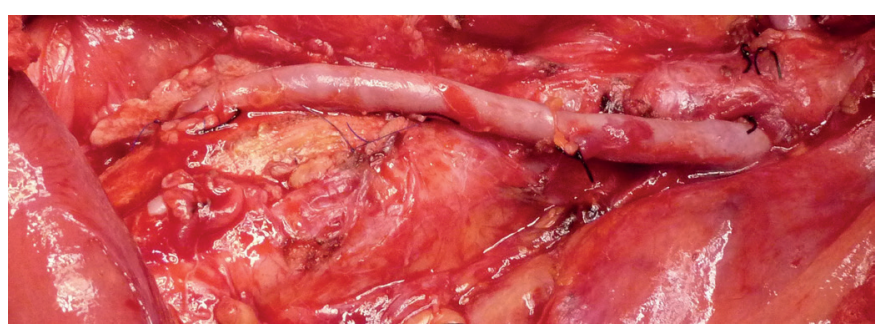

Fig. 6 Iliac-mesenteric bypass with the great saphenous vein 
polyvinyl alcohol), which is performed under local anesthesia $(9,10)$. According to literature, endovascular treatment of visceral artery aneurysms was effective in 95-98\% of cases. Reintervention was required in 3-5\% of cases. Aneurysm-related thirty-day mortality rate was 3-4\%, and the peri-procedural mortality rate was about $6 \%(3,9)$.

In our study, only $5(35.7 \%)$ patients underwent endovascular treatment. Two (14.3\%) patients underwent attempted endovascular treatment that was not effective due to tortuosity of the affected visceral artery.

Contraindications to endovascular treatment may include vascular tortuosity and the length of the affected artery, especially in case of stent implantation when there is a need to fix the proximal and distal ends (8). Complications of endovascular surgery may include thrombosis resulting in visceral ischemia, stent or coil migration, stent occlusion, reperfusion, rebleeding, nephropathy, access-related complications (femoral pseudoaneurysm, hematoma, thrombosis or embolism, infection) (13).

When it is impossible to perform endovascular treatment, surgical treatment, that involves the exclusion of aneurysmal sac, arterial bypass, vessel ligature, is recommended. Among 14 patients, surgical treatment was performed in $64.3 \%$ of the cases. In some cases, organ resection (splenectomy, colon resection) is needed $(11,16)$. Due to the constant collateral circulation between the visceral arteries, most visceral artery aneurysms can be treated by ligation or embolization. However, they cannot be applied in superior mesenteric artery aneurysms when endovascular or surgical revascularization is always mandatory (4).

The choice of treatment tactics (surgical or endovascular) for hemodynamically unstable patients is controversial. We prefer surgical treatment, although there were a few articles on successful endovascular treatment of visceral artery aneurysms in hemodynamically unstable patients $(10,17)$.

Both in the case of endovascular surgery and traditional surgery, the most serious postoperative complication is visceral ischemia that not always is acute, thereby complicating both timely diagnosis and adequate treatment (18).

In the study group, visceral ischemia as a postoperative complication after surgical treatment of superior mesenteric artery pseudoaneurysm was observed in 1 patient.

The clinical picture was changed by a specific sign of the postoperative course (changes in the trajectory of the gastrointestinal contents passage after pancreaticoduodenal resection, adhesions, the significant extent of surgery). This case confirmed that in case of severe postoperative course, even if surgery was uneventful, the presence of iatrogenic injury to the visceral arteries should be taken into account. Ultrasonography was found to play an important role in the postoperative period. If there is any fluid collection, a differential diagnosis with the detection or exclusion of an active venous or arterial blood flow is required. If there are any abnormal abdominal masses, an objective diagnostic method is CT with intravenous bolus contrast medium injection.

The choice of surgery extent (resection of superior mesenteric artery pseudoaneurysm and application of direct transverse single sutures) was substantiated by the fact that, intraoperatively, the superior mesenteric artery was sufficiently wide that allowed us to apply sutures without stenosis formation. Moreover, our goal was to minimize the extent of surgery, as, in the case of a subacute process, anastomosis or plasty may result in the development of other postoperative complications.

Superior mesenteric artery occlusion was diagnosed on the second day after surgery, however. This was most likely due to pronounced infiltrative changes in the pancreaticoduodenal region. An unfavorable prognostic criterion in the postoperative period is intestinal ischemia. Therefore, rapid recognition of the patient's clinical condition is the key to treatment success (19). If the diagnosis of mesenteric ischemia is confirmed, emergency surgery is needed (20).

We performed emergency surgery and arterial reconstruction, namely iliac-mesenteric bypass. Next day, segmental resection of the jejunum $(70-80 \mathrm{~cm})$ was performed. As it is impossible to assess the viability of the small intestine, many surgeons use minimally invasive surgical interventions (resection of the necrotic segment) and delayed, second-look surgical revision of the intestine (21). On the other hand, practically no alternative approach to surgical treatment of the patients with mesenteric ischemia exists (22). The factor of acute intestinal ischemia duration is of extreme importance in the prediction of surgical treatment success for the patients with mesenteric ischemia (23). Therefore, careful attention should be paid to clinical signs of acute intestinal ischemia, especially in the patients who underwent visceral artery reconstruction.

\section{CONCLUSIONS}

The choice of the method for treatment of visceral artery aneurysms and visceral artery pseudoaneurysms depends on the location, size, anatomic features of the visceral arteries and the clinical course of the disease. Both endovascular and surgical treatment demonstrate good postoperative outcomes.

In the case of visceral artery reconstructions, the potential risk of both the development of acute mesenteric ischemia and visceral artery occlusion should be taken into account. Visceral ischemia is one of the most serious complications in the postoperative period, which can complicate both the diagnosis and the choice of treatment tactics. In acute intestinal ischemia, emergency surgery, that involves the revascularization of the intestine, the assessment of intestinal viability and segmental resection of the necrotic intestinal segment, is required.

\section{REFERENCES}

1. Christensen T, Matsuoka L, Heestand G, et al. Iatrogenic pseudoaneurysms of the extrahepatic arterial vasculature: management and outcome. HPB (Oxford) 2006; 8(6): 458-64.

2. Regus S, Lang W. Rupture Risk and Etiology of Visceral Artery Aneurysms and Pseudoaneurysms: A Single-Center Experience. Vasc Endovascular Surg 2016; 50(1): 10-5. 
3. Jesinger RA, Thoreson AA, Lamba R, et al. Abdominal and pelvic aneurysms and pseudoaneurysms: imaging review with clinical, radiologic, and treatment correlation. Radiographics 2013; 33: 71-96.

4. Sanchez Arteaga A, Orue-Echebarria MI, Zarain L, et al. Acute bleeding from pseudoaneurysms following liver and pancreaticobiliary surgery. Eur J Trauma Emerg Surg 2017; 43(3): 307-11.

5. Cordova AC, Sumpio BE. Visceral artery aneurysms and pseudoaneurysms - should they all be managed by endovascular techniques? Ann Vasc Dis 2013; 6(4): 687-93.

6. Luckhurst CM, Perez C, Collinsworth AL, Trevino JG. Atypical presentation of a hepatic artery pseudoaneurysm: A case report and review of the literature. World J Hepatol 2016 Jun 28; 8(18): 779-84.

7. Pitton MB, Dappa E, Jungmann F, et al. Visceral artery aneurysms: incidence, management, and outcome analysis in a tertiary care center over one decade. Eur Radiol 2015; 25: 2004-14.

8. Huang YK, Hsieh HC, Tsai FC, et al. Visceral artery aneurysm: risk factor analysis, and therapeutic opinion. Eur J Vasc Endovasc Surg 2007; 33(3): 293-301.

9. Fankhauser GT, Stone WM, Naidu SG, et al. Mayo Vascular Research Center $\mathrm{C}$. The minimally invasive management of visceral artery aneurysms and pseudoaneurysms. J Vasc Surg 2011; 53: 966-70.

10. Sachdev U, Baril DT, Ellozy SH, et al. Management of aneurysms involving branches of the celiac and superior mesenteric arteries: a comparison of surgical and endovascular therapy. J Vasc Surg 2006; 44: 718-24

11. Pulli R, Dorigo W, Troisi N, et al. Surgical treatment of visceral artery aneurysms: a 25-year experience. J Vasc Surg 2008; 48: 334-42.

12. Nwafor IA, Eze JC, Ezemba N, Ngene CI, Akpan AF. Giant pseudoaneurysm of a splanchnic artery; a case report. Niger J Med 2015; 24(3): 268-72.

13. Abdelgabar A, d'Archambeau O, Maes J, et al. Visceral artery pseudoaneurysms: two case reports and a review of the literature. J Med Case Rep 2017; 11(1): 126.
14. Habib N, Hassan S, Abdou R, et al. Gastroduodenal artery aneurysm, diagnosis, clinical presentation, and management: a concise review. Ann Surg Innov Res 2013; 7: 4.

15. Volpi MA, Voliovici E, Pinato F, et al. Pseudoaneurysm of the gastroduodenal artery secondary to chronic pancreatitis. Ann Vasc Surg 2010; 24: 1136.e1137-1111.

16. Arca MJ, Gagner M, Heniford BT, et al. Splenic artery aneurysms: methods of laparoscopic repair. J Vasc Surg 1999; 30: 184-88.

17. Dohan A, Eveno C, Dautry R, et al. Role and effectiveness of percutaneous arterial embolization in hemodynamically unstable patients with ruptured splanchnic artery pseudoaneurysms. Cardiovasc Intervent Radiol 2015; 38: 862-70.

18. Morbi AH, Nordon IM. Emergency revascularisation in a patient with acute mesenteric ischemia: the role of open revascularisation and compensatory blood flow. Acta Chir Belg 2016; 116(4): 234-38.

19. Savlania A, Tripathi RK. Acute mesenteric ischemia: a current multidisciplinary approach. J Cardiovasc Surg (Torino) 2017; 58(2): 339-50.

20. Renner $\mathrm{P}$, Kienle $\mathrm{K}$, Dahlke $\mathrm{MH}$, et al. Intestinal ischemia: current treatment concepts. Langenbecks Arch Surg 2011; 396(1): 3-11.

21. Acosta S. Surgical management of peritonitis secondary to acute superior mesenteric artery occlusion. World J Gastroenterol 2014; 20(29): 9936-41.

22. Oderich GS, Macedo R, Stone DH, et al. Multicenter study of retrograde open mesenteric artery stenting through laparotomy for treatment of acute and chronic mesenteric ischemia. J Vasc Surg 2018; 68(2): 47-480.e1.

23. Ding W, Wang K, Liu B, et al. Open Abdomen Improves Survival in Patients With Peritonitis Secondary to Acute Superior Mesenteric Artery Occlusion. J Clin Gastroenterol 2017; 51(9): e77-e82. 